\title{
Numerical Stress Intensity Factor in Polymer Structures
}

\author{
R. RHANIM*, R. EL HAIMER**, Y. TOUNSI*, H. RHANIM**, A. NASSIM* \\ *Laboratoire Instrumentation de Mesure et de Contrôle IMC, Physics Department, Chouaïb Doukkali University, \\ d'EL Jadida, Maroc, E-mail: rhanim.h@ucd.ac.ma \\ **Laboratoire de Mécanique et Energétique LME, Physics Department, Chouaïb Doukkal University, \\ d'EL Jadida, Maroc
}

cross $^{\text {ref }}$ http://dx.doi.org/10.5755/j01.mech.24.2.17329

\section{Introduction}

Because of their severe operational conditions, many industrial components are subject to complex combinations of mechanical constraint. These combinations are at the origin of initiation and propagation of cracks in these parts, which contributes to the ruin of the structure [1].

Thus, the knowledge of the maintenance of these parts under such conditions becomes essential because it enables us to predict their lifetime. The purpose of this study is, first of all, to determine the concentration of the constraints in the vicinities of the defects by the stress intensity factor FIC in a part submitted to a loading of traction.

Linear fracture mechanics is based on an analytical procedure that connects the stress field in the vicinity of the crack, the nominal stress applied in the distance to the crack size and orientation and finally to the mechanical characteristics of the material. Various analytical methods are used to study the fields of displacements, strains and stresses in the vicinity of a crack $[2,3]$.

The adopted Approach is the energy approach which is based on the energy analysis of the continuous medium containing a crack. It is about a total energy assessment integrating the rate of refund of energy due to a virtual increase in the crack by the determination of J-integral.

\section{Study design}

In this study, three types of specimens were used. The first was a perforated specimen subjected to uniaxial tensile stress. Stress concentration factor Kt due to the presence of the hole was determined numerically by the finite element method. The second and third structures are specimens of the same geometry as the first and respectively with a notch and double notches starting from the hole, subjected to uniaxial tensile loading $(6 \mathrm{~N})$. Following the study was devoted to the numerical determination of the stress intensity factor SIF.

The dimensions of the developed geometrical model are 160x40x5 mm. The hole diameter is $20 \mathrm{~mm}$ for the notches having a length of $1 \mathrm{~mm}$ (Fig. 1).

Numerical simulation was made by ABAQUS computer code. The specimen is meshed with S3 type of triangular elements with a total number of elements equal to 12312. A mesh refinement is also set in the neighborhood of the default (hole), to detect the concentration of local stresses in this area, and give more relevance to results (Fig. 2).

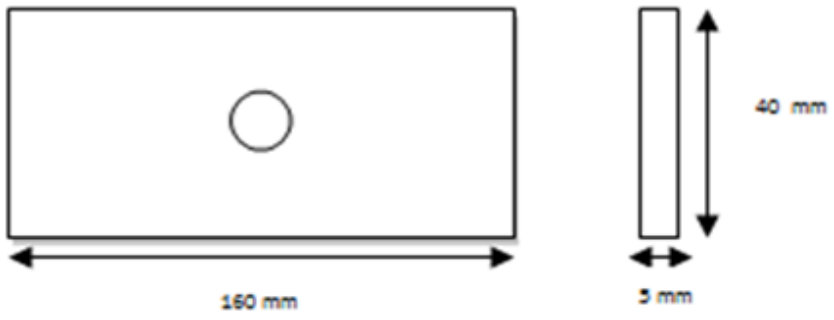

Fig. 1 Specimen dimensions

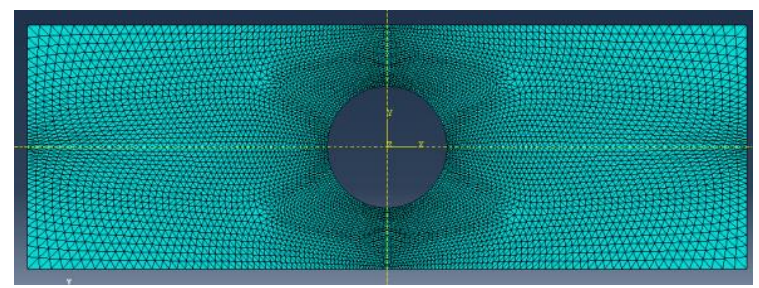

Fig. 2 Representation of a meshed specimen

The specimen is a transparent polymer considered elastic Young's modulus of $E=2.7 \mathrm{~N} / \mathrm{mm}^{2}$ and Poisson's ratio $v=0.48$.

\section{Results and discussions}

\subsection{Stress distribution}

The results shown in the Fig. 3 below shows the distribution of stresses in specimen with hole under a tensile loading.

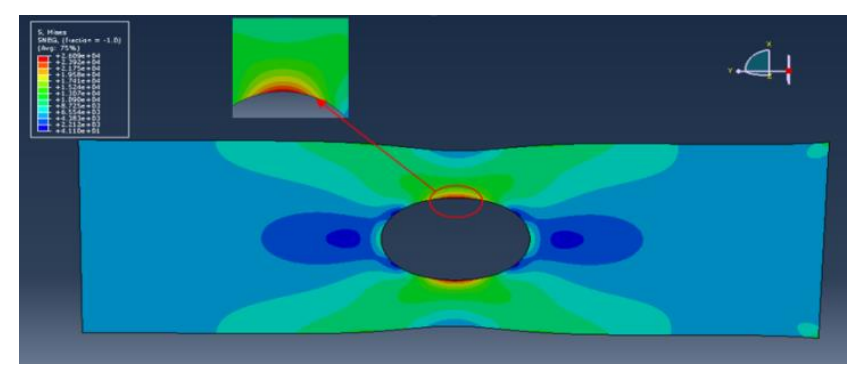

Fig. 3 The distribution of stresses in specimen with hole under a tensile loading

It is clear that the loading Applied to the structure generated the maximum stresses in the vicinity of the hole. Fig. 4 illustrates the stress distribution along a predefined path. 


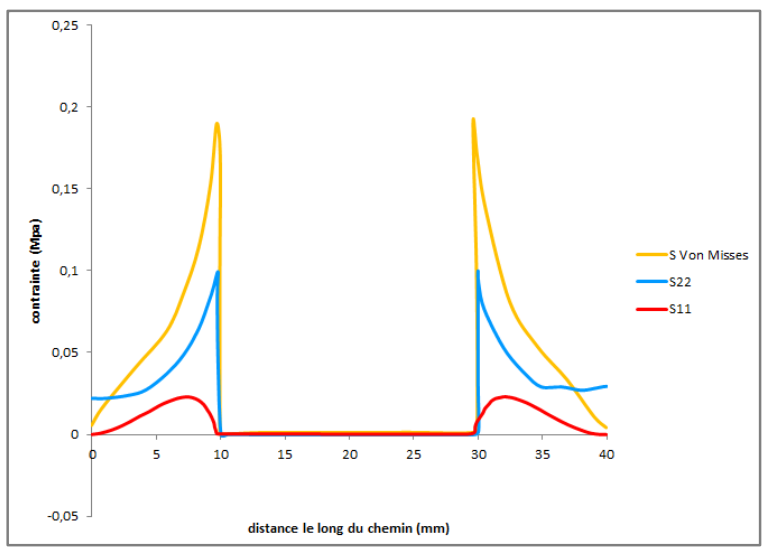

Fig. 4 The stress distribution along a predefined path

3.2. Effect of single and double a notch on the specimen damage

In this part we are interested in modeling the structure with single and double notch to highlight the influence of the crack on the behavior of specimens. Figs. 5 and 6 illustrate the distribution of equivalent stresses induced locally around defects.

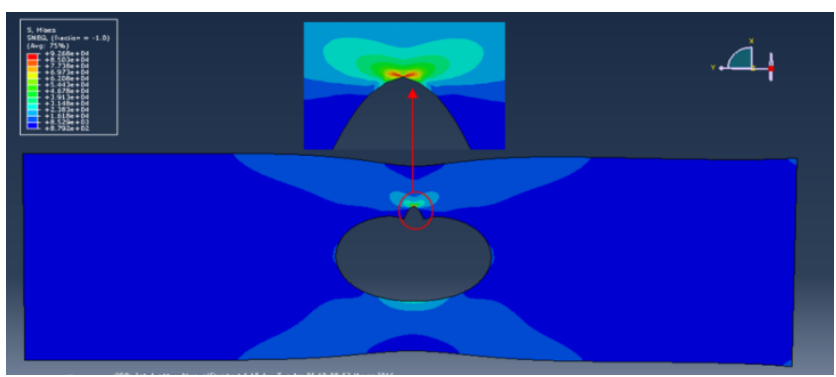

a

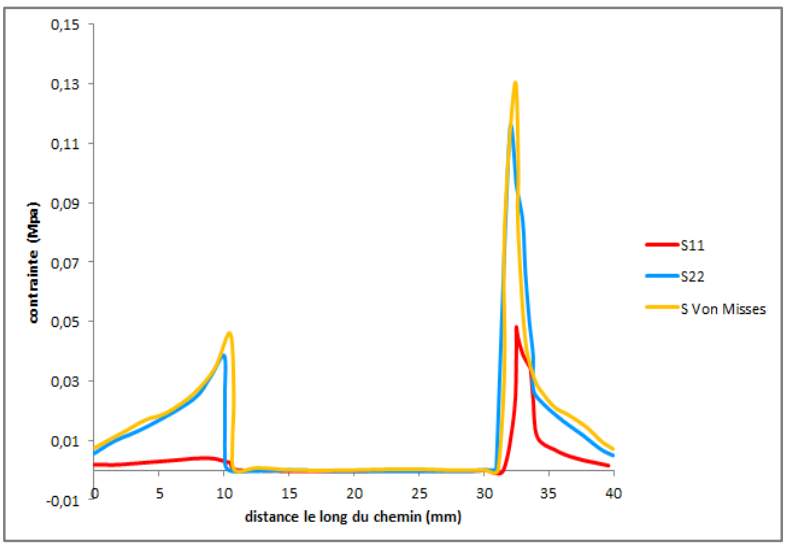

b

Fig. 5 The distribution of stresses induced locally around defects in single notched specimen (a). The stress distribution along a predefined path (b)

Disturbance of the shape of the test piece by performing notch causes a discontinuity in the material and consequently a stress distribution compared to that of a healthy breakthrough test. This is called stress concentrations are highest at the bottom of the notch. We also noted that the presence of a single notch generates higher stress concentration than the double notch.

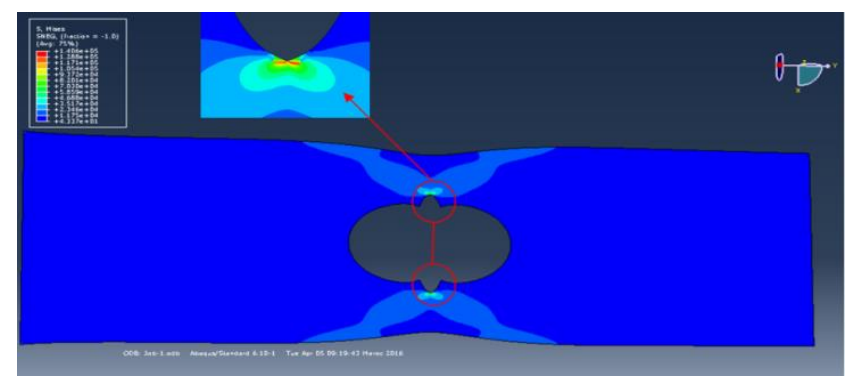

a

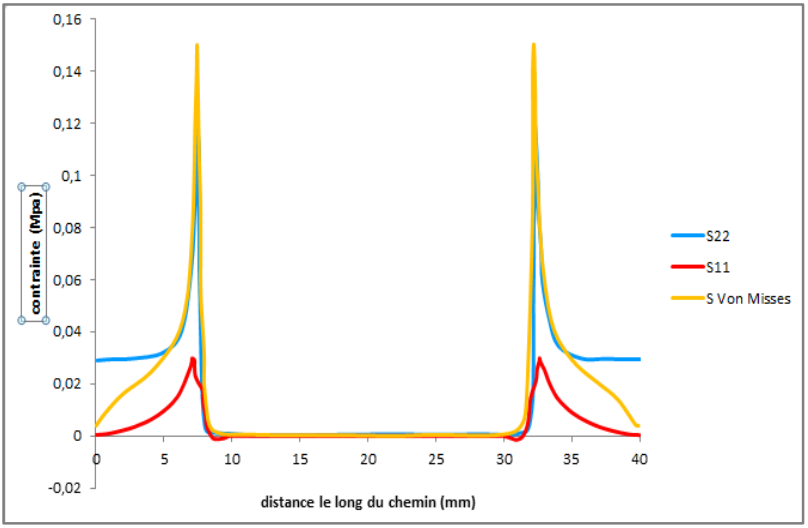

b

Fig. 6 The distribution of stresses induced locally around defects in double notched specimen (a). The stress distribution along a predefined path (b)

\subsection{Stress intensity factor}

This part of the manuscript we are interested to evaluating the stress intensity factor by the method of finite elements integrated in Abaqus computer code. Calculating the FIC based on the determination of the energy $\mathrm{J}$ required for the crack can propagate. The method used is that contour integrals.

The J-integral represents a way to calculate the strain energy release rate, 1 [4]. The theoretical concept of $J$ integral was developed in 1967 by Cherepanov [5] and in 1968 by Jim Rice [6] independently, who showed that an energetic contour path integral (called $J$ ) was independent of the path around a crack.

The SIF are defined from the energy release rate $J$. The $J$-integral around a crack tip is frequently expressed in a more general form as:

$$
J=\int_{\Gamma} W d y-\vec{T} \frac{\overrightarrow{\partial v}}{\partial x} d s,
$$

where: $\Gamma$ is closed contour surrounding the crack; $d s$ is contour element; $W$ is strain energy density: $T$ is surface stress vector; $v$ is displacement vector.

The stress intensity factor, under the assumption of plane stresses and in the presence of a homogeneous isotropic material and in mode $\mathrm{I}$ is connected to $\mathrm{J}$ by the following relationship:

$$
J=\frac{K_{I}^{2}}{E},
$$


where: $K_{I}$ is SIF under mode $I ; E$ is Young modulus.

A refinement of mesh was carried out in the neighborhoods of the crack tip in order to give more precision to the results.

The values of the $J$-integral on different contours are presented in the following Figs. 7 and 8.

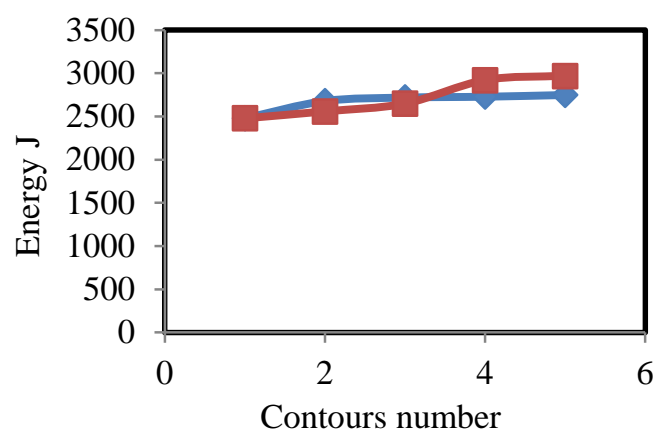

Fig. 7 Value of the $J$-integral in the specimen doubly notched

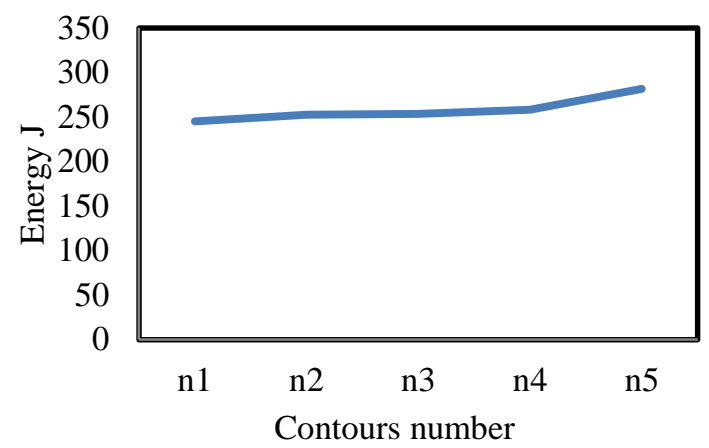

Fig. 8 Value of the $J$-integral in the specimen simply notched

In theory, the $J$-integral is independent of the selected contour. However, the calculation results obtained for the case of simply and doubly notched specimen show that from the second contour, the value of $J$ converges to a somewhat constant value nearly all contours.

\subsubsection{SIF calculation}

The stress intensity factor of simply and doubly notched specimens is determined from energies (J-integral) previously determined using the following formula:

$$
K_{I}=\sqrt{J E} \text {. }
$$

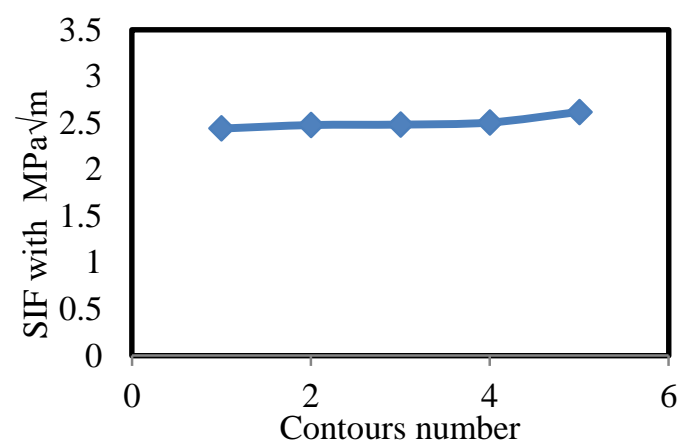

Fig. 9 Value of the SIF in the specimen simply notched

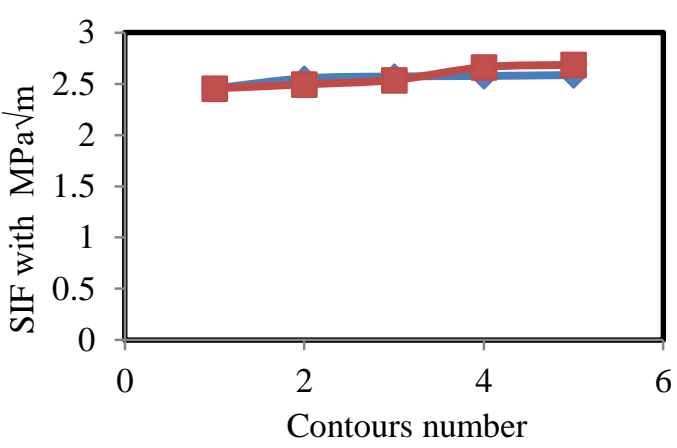

Fig. 10 Value of the $J$-integral in the specimen doubly notched

\section{Photoelasticity technique}

\subsection{Introduction}

Photoelasticity is a well-established technique for stress analysis, and it has a wide range of industrial and research applications [7]. Several methods of analyzing photo elastic fringe patterns by means of phase-measuring techniques have been presented [8-10]. The principal stress difference can be easily obtained by phase extraction algorithm. There are many methods [11] to extract phases from fringe patterns such as Phase shifting technique, Wavelets demodulation [12], Spiral transform [13] and Monogenic signal [14].

The monogenic signal is two-dimensional generalization of the analytic signal proposed by Felsberg and Sommer [15]. The monogenic signal for an image is defined as the combination of the image and its Riesz transform components. The Riesz Transform [16] is a generalization of Hilbert Transform. The monogenic signal is characterized by its three independent local sizes as amplitude, phase vector, and orientation.

\subsubsection{Photoelastic fringe pattern intensity}

The experimental set up called polariscope is composed by polarizer $P$, analyzer $A$ and two quarter wave plates $Q . R$ stand for stressed model (retarder). Therefore, by $P 90 Q 45 R \alpha, \phi Q \beta A \gamma$, we mean; the polarizer at $90^{\circ}$, the first quarter-wave plate with fast axis at $45^{\circ}$, the specimen as retardation $\phi$ and whose fast axis is at an angle $\phi$ with the $\mathrm{x}$-axis, the second quarter-wave plate with fast axis at $\beta$, and the analyzer at $\gamma$ (Fig. 11).

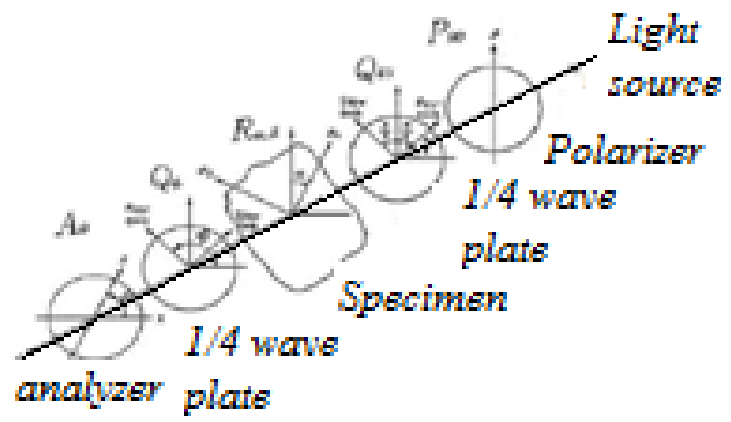

Fig. 11 Optical arrangement of a circular polariscope 
The obtained intensity distribution is:

$$
\begin{aligned}
& I=i_{m}-i_{v}(\sin 2(\gamma-\beta) \cos \phi- \\
& -\sin 2(\beta-\alpha) \cos 2(\gamma-\beta) \sin \phi),
\end{aligned}
$$

where: $i_{m}$ is the bias (stray light term), $i_{v}$ is visibility function (the constant of proportionality). When the wave is circularly polarized, the quarter wave plates are positioned after the polarizer and the fast and slow axes of the plates are at $45^{\circ}$ with respect to polarizer axis, and the two quarter wave plates are crossed followed by the analyzer. The fringe pattern intensity for circular configuration $P 90 Q 45 R \alpha, \phi Q 0 A 45$ is:

$$
I=i_{m}-i_{v} \cos \varphi
$$
ference by:

The phase is related to the variation of the path dif-

$$
\phi=\frac{2 \pi \delta}{\lambda}=2 \pi \frac{(e \Delta n)}{\lambda},
$$

where: $\lambda$ is the wavelength; $\delta$ is the path difference; $e$ is the thickness of the material and $\Delta n$ is the refractive index variation. The phase distribution can be expressed using the principal stress difference $\Delta \sigma$ and the photoelastic constant $C$ which characterize the specimen as:

$$
\phi=2 \pi \frac{(C e \Delta \sigma)}{\lambda} .
$$

We easily obtain the principal stress difference by phase extraction algorithm. In this work, the phase is extracted from phase vector of the monogenic signal.

The phase vector can be interpreted as a rotation vector, which magnitude corresponds to the phase angle between the image and the monogenic signal. When the monogenic signal is applied to a fringe pattern, the phase vector gives the optical phase distribution of fringes.

$$
\phi=\frac{q_{2}}{\left(q_{1}^{2}+q_{2}^{2}\right)^{1 / 2}} \arctan \frac{\left(q_{1}^{2}+q_{2}^{2}\right)^{1 / 2}}{p} .
$$

In order to get continuous phase distribution, the values at the phase jump are connected by means of unwrapping procedure [17].

\section{2. Experimental results}

In Fig. 12, we present the experimental set up. Fig. 13, a illustrate photoelasticimetry fringe pattern. Fig. 13, b shows the wrapped phase distribution obtained from the monogenic signal. Fig. 13, c shows the unwrapped phase by PUMA algorithm.

Note that the distribution of the equivalent stress in the specimen marks a maximum stresses concentration in the vicinity of the defect (hole), while away from defect there are low stress values. Far from the discontinuity specimen is relaxed.

These results are in good agreement with those found by the numerical method.

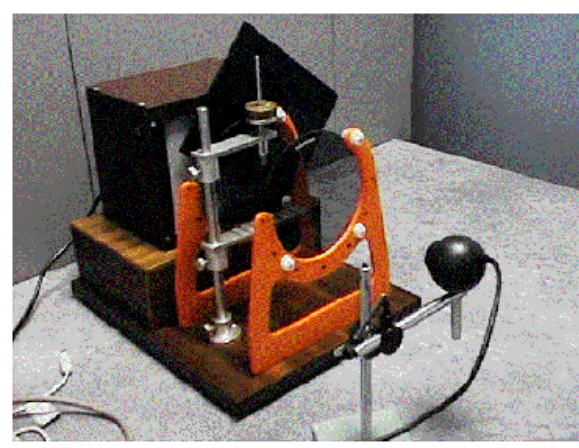

Fig. 2 Experimental set up

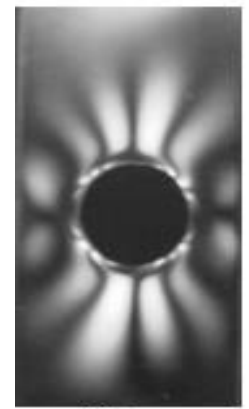

(a)

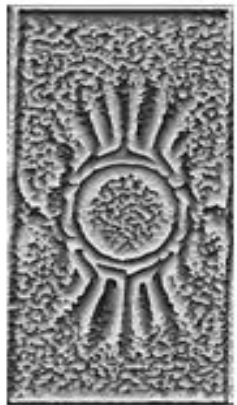

(b)

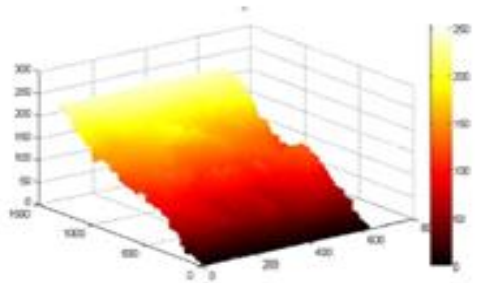

(c)

Fig. 13 Isochromatic fringe pattern (gray scale) (a), wrapped phase distribution obtained using the monogenic signal (b), continuous phase distribution (c)

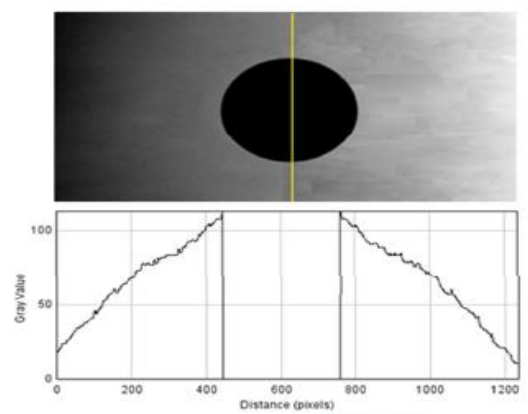

Fig. 14 Intensity distribution along the path in continuous phase distribution

\section{Conclusion}

This communication was like to study using the finite element method the distribution of stresses induced in structures with discontinuities. These structures are specimens with hole and respectively with single notch and double notch. The results obtained in the light of this first part of study are:

- Distribution of the equivalent stress in the specimen mark maximum stress concentration in the vicinity of defects (hole and notches), while away from the default there 
is low stress values. Stress concentration is sensitive to the type of default.

- Comparing the maximum values of equivalent stress for three simulated specimens is noted that the test hole with a simple notch is the one with the highest value. On the basis of this study we conclude that the simple cut is the most critical defect which gives the shorter life time structure relative to other structures and consequently will lead quickly to the brutal destruction of the specimen.

The second part of this work was devoted to the determination of the stress intensity factor by the energy approach adopted by the Abaqus computer code. The SIF was calculated in the mode I, a contour number five, the $J$-integral approach in both samples which led to obtaining value pretty constant meadows.

Finally, the principal stress distributions are determined from the optical phase distribution by using bidimensional photoelasticimetry technique. The experimental results are compared to numerical finite element study.

The good concordance of the results allows us to hope the application of this technique to more complex configurations.

\section{References}

1. G'sell, C. 2002. Plasticité et endommagement des polymères structuraux, Matériaux de structures, 40-43.

2. Kausch, H. 2005. intrinsic mobility and toughness of polymers, Springer verlog 1-35. http://dx.doi.org/ 10.1007/b136948.

3. Dermici, I. 2004. Mécanique de l'endommagement par rayure de polymères, thèse de doctorat, ULP strasbourg. Available from internet: http://scd-theses.u-strasbg.fr/.

4. Van Vliet, Krystyn J. 2006. 3.032 Mechanical Behavior of Materials, available from internet: https://ocw.mit.edu/courses/materials-science-and-engineering/3-032-mechanical-behavior-of-materials-fall2007/lecture-notes/lec1.pdf.

5. Cherepanov, G. P. 1967. Crack propagation in continuous media: PMM, Journal of Applied Mathematics and Mechanics 31(3): 503-512. http://dx.doi.org/10.1016/0021-8928(67)90034-2.

6. Rice, J. R. 1968. A Path Independent Integral and the Approximate Analysis of Strain Concentration by Notches and Cracks, Journal of Applied Mechanics 35: 379-386. http://dx.doi.org/10.1115/1.3601206.

7. Frocht, M. M. Wiley, New York, 1941 and 1948. Photoelasticity.

8. Asundi, A. 1993. Phase shifting in photoelasticity, Exp. Tech. 17: 19-23. http://dx.doi.org/10.1111/j.1747-1567.1993.tb00269.x.

9. Buckberry, C.; Towers, D.1995. Automatic analysis of isochromatic and isoclinic fringes in using phase measuring techniques, Meas. Sci. Technol. 6: 1227-1235. http://dx.doi.org/10.1088/0957-0233/6/9/001.

10. Afifi, M.; Fassi, F. A.; Marjane, M.; Nassim, K.; Sidki, M.; Rachafi, S.2002. Paul wavelet based algorithm for optical phase distribution evaluation, Opt Commun 211: 47-51.
http://dx.doi.org/10.1016/S0030-4018(02)01828-X.

11. Creath, K. 1998. Phase - measurement interferometry techniques, In Wolf E (ed.): Progress in optics Vol. XXVI. 349-393 Elsevier Science, (Amsterdam). http://dx.doi.org/10.1016/S0079-6638(08)70178-1.

12. Quiroga, J. A.; Gonzalez-Cano, A. 1997. Phase measuring algorithm for extraction of isochromatics of photoelastic fringe patterns, Appl. Opt. 36: 8397-8402. https://doi.org/10.1364/AO.36.008397.

13. Larkin, K. G.; Bone, D. J.; Oldfield, M. A. 2001. Natural demodulation of two- dimensional fringe patterns. I General background of the spiral phase quadrature transform, J. Opt. Soc. Am. A 18: 1862-1870. https://doi.org/10.1364/JOSAA.18.001862.

14. Felsberg, M.; Sommer, G. 2003. The monogenic scalespace: A unifying approach to phase-based image processing in scale-space, J. Math. Imag. Vision 21:5-26. https://doi.org/10.1023/B:JMIV.0000026554.79537.3 5.

15. Felsberg, M.; Sommer, G. 2001. The monogenic signal, IEEE Transactions on Signal Processing 49(12): 3136-3144. https://doi.org/10.1109/78.969520.

16. Riesz, M. 1928. Sur les fonctions conjuguées, Mathematische Zeitschrift, vol. 27, no. 1, 218-244. http://eudml.org/doc/167977. https://doi.org/10.1007/BF01171098.

17. Schofield, M. A.; Zhu, Y. 2003. Fast phase unwrapping algorithm for interferometric applications, Optics Letters 28(14). https://doi.org/10.1364/OL.28.001194.

\author{
R. Rhanim, R. EL Haimer, Y. Tounsi, H. Rhanim, \\ A. Nassim
}

\section{NUMERICAL STRESS INTENSITY FACTOR IN POLYMER STRUCTURES}

S u m m a r y

The purpose of this study was to determine the SIF stress intensity factor by numerical modeling of a structure containing a hole playing the role of a discontinuity "crack" subjected to a tensile load by the $J$-integral approach. The energy release rate was defined by modeling the crack by a notch and a double notch starting from the hole. The results were validated experimentally by testing a transparent material using the photoelasticity technology.

Keywords: stress intensity factor, finite element method $J$-integral, two-dimensional photoelasticity measurement, monogenic signal.

Received January 23, 2017 Accepted April 18, 2018 